\title{
BIOMOLECOLE DA BASIDIOMICETI COME SORGENTE DI POTENZIALI NUOVI FARMACI
}

\author{
Nota del s.c. GIANLUCA NASINI (*)
}

(Adunanza del 19 gennaio 2012)

\begin{abstract}
SuNTO. - Sono riportati l'isolamento e la struttura di alcuni composti, biogeneticamente correlati, prodotti in coltura da vari ceppi di Basidiomiceti appartenenti ai generi Armillaria, Laurilia, Clitocybe e Clavicorona. I metaboliti isolati sono sesquiterpenoidi contenenti l'anello protoilludanico e sono stati anche correlati con l'attività biologica e schemi biosintetici. Abbiamo analizzato la chimica dei melleolidi, sulcatine, tsugicoline e clavilattoni. In particolare, la spirolaxina, isolata da colture di Sporotrichum laxum, è attiva sul batterio Helicobacter pylori ed è un potente inibitore dell'angiogenesi. La sua struttura è stata determinata con evidenze chimiche e spettroscopiche e confermata mediante analisi con $\mathrm{i}$ raggi $\mathrm{X}$.
\end{abstract}

$* * *$

ABSTRACT. - We reported the isolation and structural characterization of a new biogenetical related compounds, produced in cultures by various strains of Basidiomycetes of the genera Armillaria, Laurilia, Clitocybe and Clavicorona. The isolated metabolites are sesquiterpenoids containing the protoilludane ring system and are even correlated with some biological activity and biogenetic steps. We have analyzed the chemistry of the melleolides, sulcatines, tsugicolines and clavilactones. In particular, the spirolaxine, isolated from cultures of Sporotrichum laxum, is active on the bacterium Helicobacter pylori and is a potent inhibitor of angiogenesis. Its structure was determined on the basis of chemical and spectroscopic evidence and confirmed by X-ray analysis.

(*) CNR-Istituto di Chimica del Riconoscimento Molecolare. Dipartimento di Chimica, Materiali ed Ingegneria Chimica del Politecnico, Via Mancinelli 7, Milan, Italy. E-mail: gianluca.nasini@polimi.it 


\section{INTRODUZIONE}

Scopi di questa ricerca sono stati la coltivazione del micelio, l'isolamento, la determinazione della struttura, la stereochimica, le correlazioni chimiche di nuove sostanze e lo studio dell'attività biologica di nuovi metaboliti prodotti da Basidiomiceti; inoltre la possibilità di coltivare il fungo su diversi substrati aumenta la possibilità di isolare nuovi metaboliti, sfruttando il ricco patrimonio enzimatico del fungo stesso.

Una serie di nuovi sesquiterpeni di origine protoilludanica sono stati isolati da funghi dei generi, Armillaria, Laurilia, Clitocybe e Clavicorona.

Nel nostro progetto sono stati studiati inizialmente i metaboliti prodotti dal fungo Armillaria mellea, un basidiomicete che è noto produrre sostanze che possiedono attività antibatterica; abbiamo isolato su terreno GYA (glucosio-lievito-agar) i melleolidi B-H mentre su terreno contenente MPG (malto-peptone-glucosio), si sono ottenuti i melledonali B-D; questi composti, che hanno una insolita idrossilazione al C-10 e C-13 sono nuovi membri della classe dei sesquiterpeni protoilludanici O-metilorsellinati (Fig. 1). Le loro strutture sono state determinate sulla base di correlazioni chimiche e dati spettroscopici. Le configurazioni assolute sono state ottenute mediante cristallografia a raggi $\mathrm{X}$ e procedure NMR.[1]

\section{Sesquiterpeni $D^{2,3}$ - protoilludanici arilesteri da Armillaria mellea}

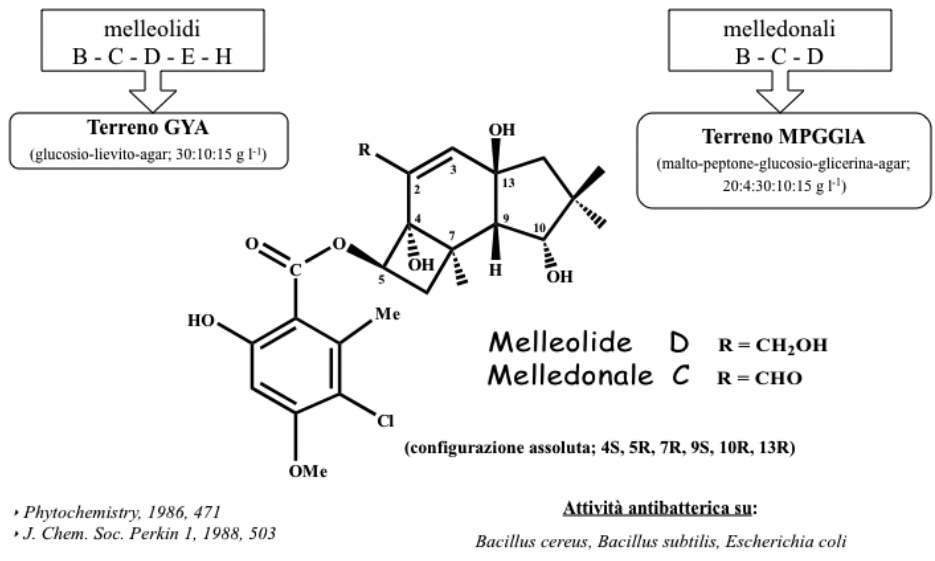

Fig. 1. 


\section{Cenere: Laurilia ; Clavicorona}

Cinque nuovi sesquiterpeni, le sulcatine A-E sono state isolate da colture liquido-stazionario (MPGB) di Laurilia sulcata (Stereum sulcatum) (Fig. 2); il ceppo proviene dalla micoteca di Baarn (CBSOlanda) e le strutture sono state risolte da misure spettroscopiche e correlazioni HETCOR e COLOC. Le strutture delle sulcatine C-E sono insolite nei sesquiterpeni di origine protoilludanica; l'apertura dell'anello a quattro termini nel co-metabolita sulcatina $\mathrm{A}$ può portare ad una reazione retroaldolica con l'apertura dell'anello ciclobutanico dando luogo ad una nuova serie di sesquiterpeni che chiameremo nor-isoilludalani (vedi schema biosintetico) (Fig. 3).[2]

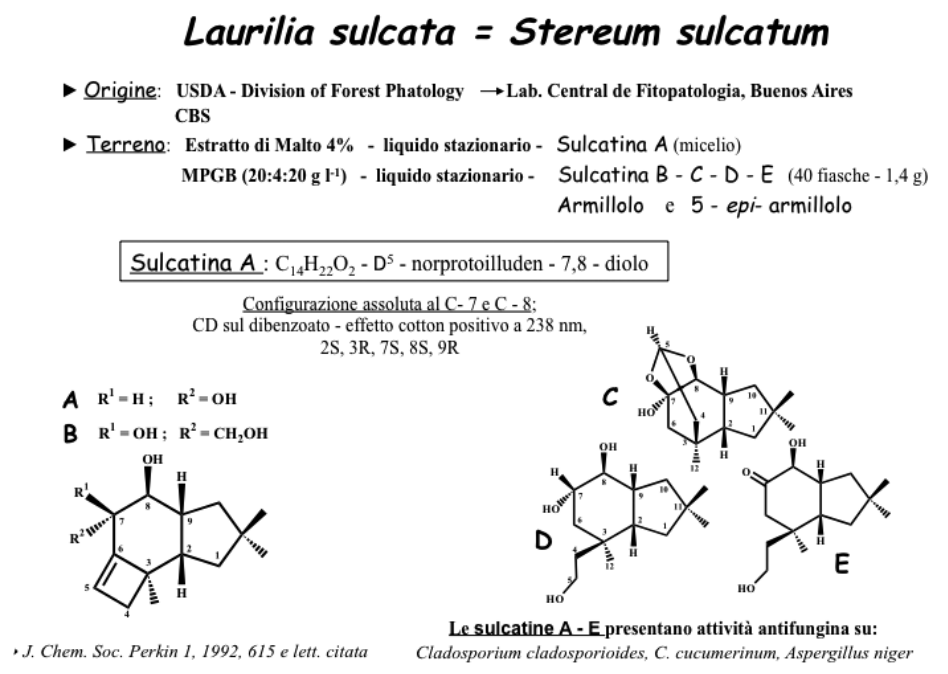

Fig. 2.

Le tsugicoline A-D (1a, 2, 3a, 4 ) provengono da fermentazione di Laurilia tsugicola su terreno MPGB (Fig. 4); in particolare 1a è un precursore biogenetico per ottenere altri sesquiterpeni con diversi scheletri; la presenza anomala di una funzione carbonilica nell'anello ciclobutanico, è la chiave della reattività di questo metabolita che 
porta ad una facile apertura del legame 6-7 con la formazione di una larga serie di nuovi metaboliti.[3]

La tsugicolina A riarrangia a $\mathrm{pH} 13$ nel composto 8 a con una struttura simile ai sesquiterpeni isolattarani e nel composto 9a come la sostanza naturale tsugicolina $\mathrm{H} 9 \mathrm{c}$; in condizioni basiche differenti, si formano i composti 4a, 5, 6a e 7. L'isolamento della sostanza 2 è il primo esempio di una conversione chimica di un protoilludano in un sesquiterpene sterpuranico (Fig. 5).[4]

\section{Schema biosintetico di formazione dei principali sesquiterpeni da Basidiomiceti}

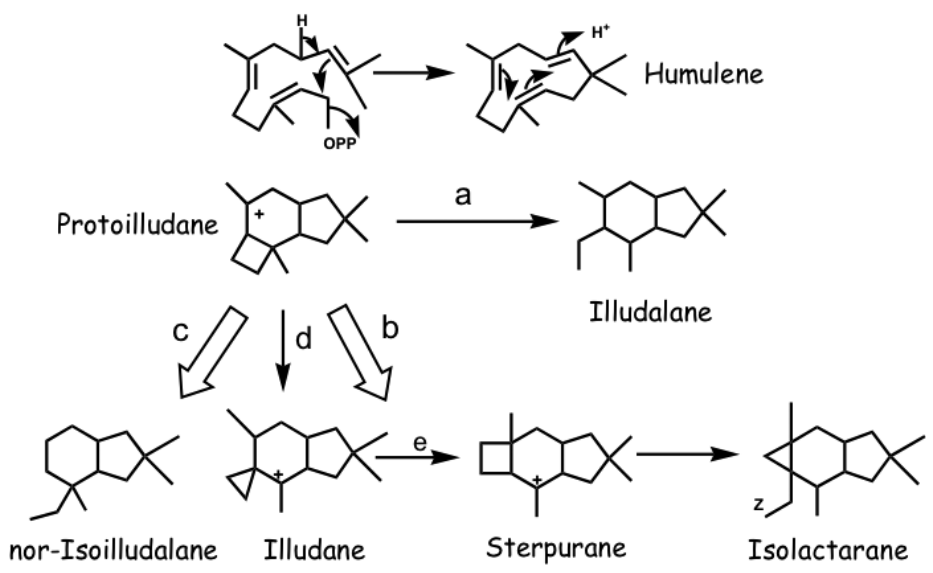

Fig. 3.

Dal fungo Clavicorona divaricata (Fig. 6) in coltura agarizzata a base di malto-peptone-glucosio (MPG) sono stati isolate principalmente le divaricatine A 3 a e B $3 b$ insieme alle tsugicoline $\mathrm{L} 2 \mathrm{~b}$ e $\mathrm{M} 5 \mathrm{~b}$ a struttura furo-sesquiterpenoidica; le loro strutture sono state determinate con l'aiuto di studi NMR. Tutti i metaboliti sono attivi contro alcuni batteri e inibiscono la germinazione di Lepidum sativum.[5] 


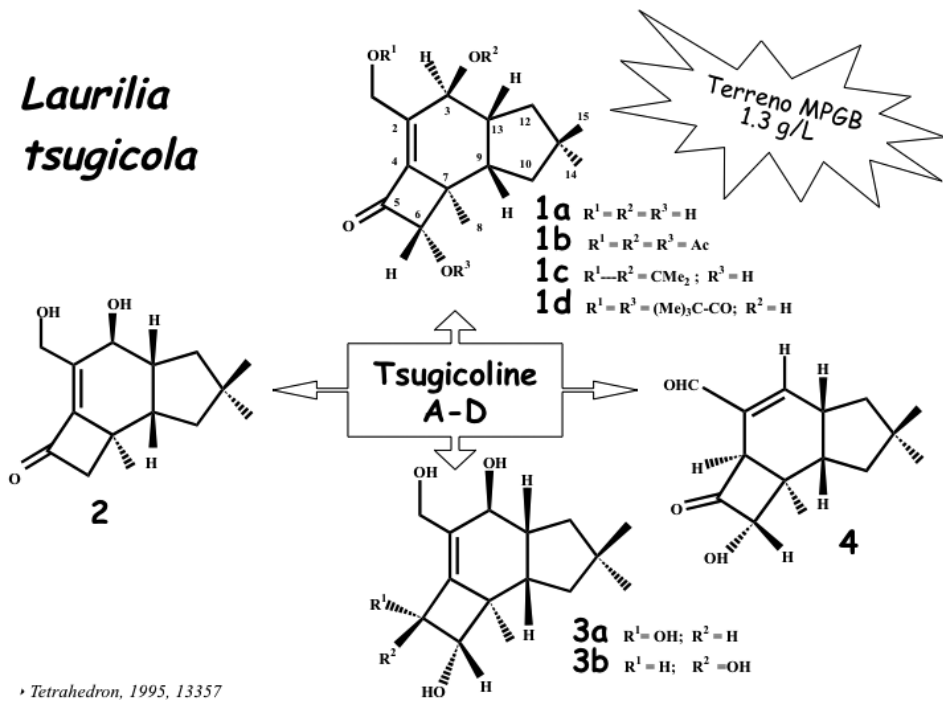

Fig. 4.

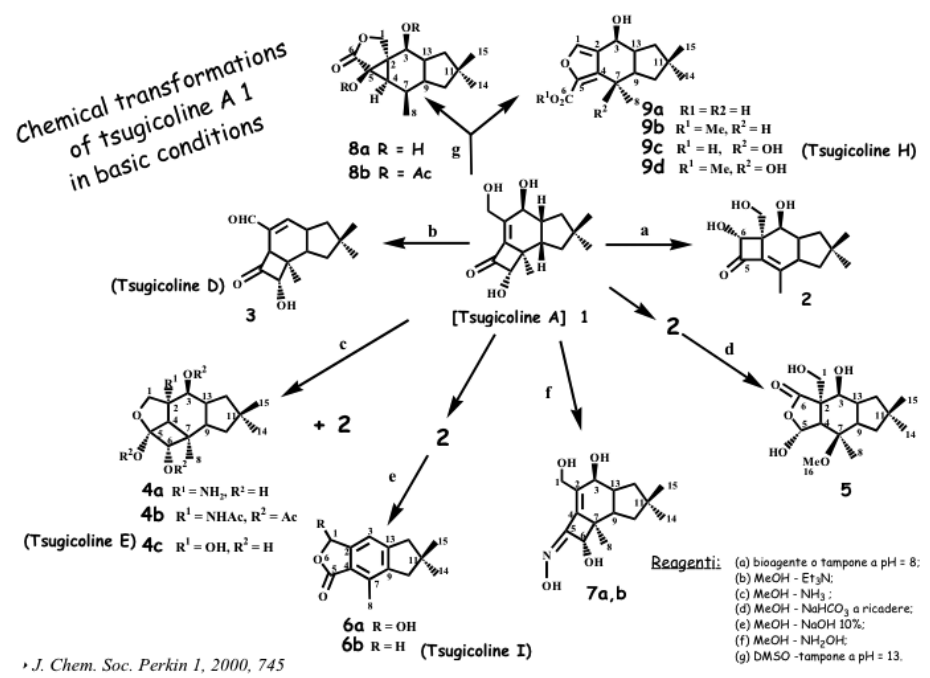

Fig. 5. 


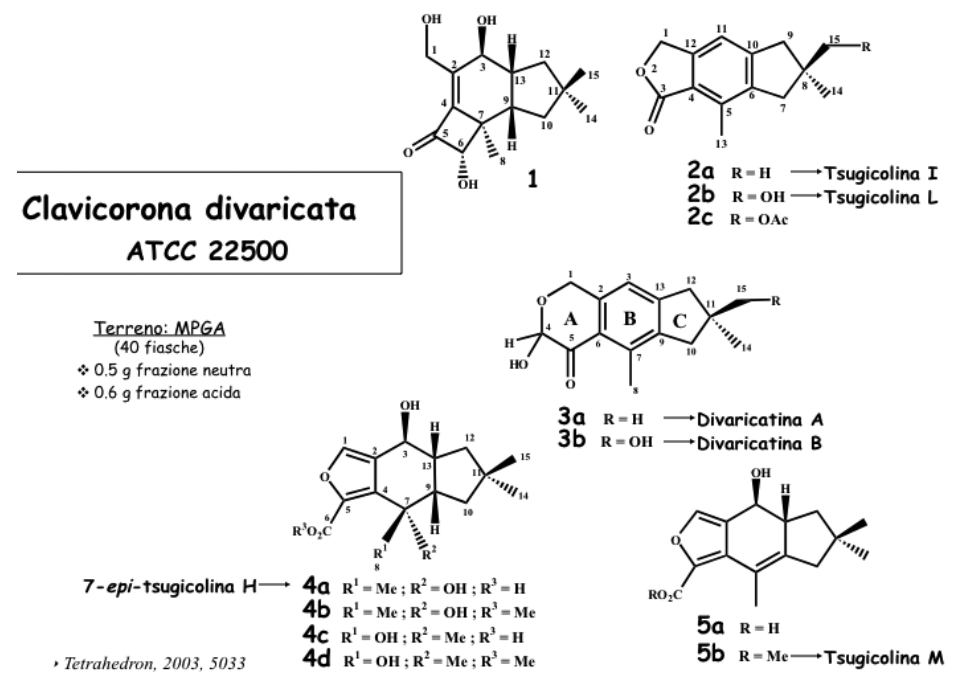

Fig. 6.

\section{Genere: CLITOCyBE ; SPOROTRICHUM}

La forte tossicità della illudina M 1 naturale (Clitocybe illudens) porta ad escludere il suo uso come agente antitumorale; allo scopo di ridurre la sua tossicità che si ritiene sia correlata alla capacità del doppio legame coniugato di dare reazioni di tipo Michael, il metabolita è stato fatto reagire con diazometano ottenendo i prodotti 2-4 mentre per ossidazione con m-CPBA è stato ottenuto il composto $5 \mathrm{a}$, questi sono risultati meno attivi del loro precursore (Fig. 7). Nella tabella sono mostrate le tossicità di questi derivati se testati sulla linea cellulare A 2780.[6]

Altri interessanti metaboliti, i clavilattoni A-E sono stati isolati da colture di Clitocybe clavipes; il clavilattone D ha mostrato inibire la proteina tyrosine kinasi.[7]

La spirolaxina 1, isolata dal fungo Sporotrichum laxum (Figg. 8,9), è un metabolita contenente un nucleo della 3-metossi-5-idrossi-ftalide legato con una catena metilenica ad un gruppo spiroacetalico; la sua stereochimica è stata determinata mediante cristallografia a raggi $\mathrm{X}$ e da misure di CD. La spirolaxina mostra attività sul batterio Helicobacter 
pylori, responsabile di ulcere gastriche; insieme al suo co-metabolita sporotricale 2 risulta anche essere un potente inibitore dell'angiogenesi. L'attività della spirolaxina viene rilevata in vitro su cellule endoteliali e su di una varieta di linee cellulari tumorali. Si è dimostrato che ad una dose non tossica (1-0.1 $\mu \mathrm{M})$, inibisce la crescita delle cellule endoteliali da microcircolo di surrene bovino (BMEC) e delle cellule endoteliali umane (UVEC); dopo 24-48 h con dosi da 1 a $40 \mu \mathrm{M}$ la molecola determina inibizione della proliferazione sulle linee tumorali MESSA (sarcoma uterino), LoVo e HL60. La sintesi della (+)-spirolaxina metiletere chiude la ricerca su questo interessante metabolita. $[8,9]$

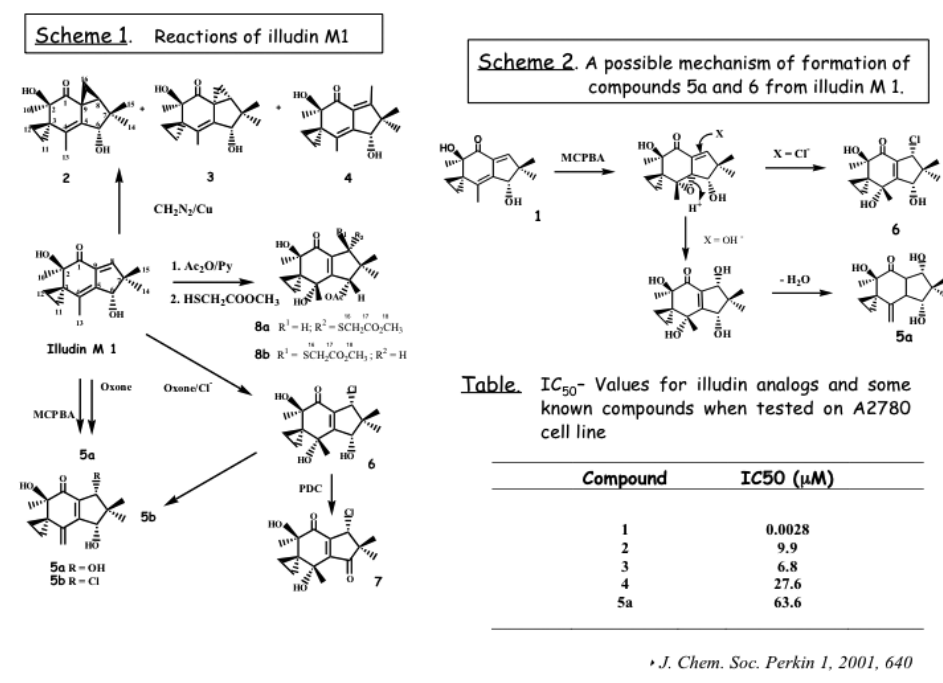

Fig. 7.

\section{I Clavilattoni}

I clavilattoni A-E sono metaboliti prodotti dal Basidiomicete Clitocybe clavipes su colture agarizzate (MPGA); questi metaboliti hanno attività antibatterica ed antifungina ed inoltre inibiscono la crescita di Lepidium sativum. 
Il fungo su di un diverso terreno (GMYA) produce il clavilattone $\mathrm{D}$ un metabolita rosso la cui struttura è stata determinata mediante studi ${ }^{1} \mathrm{He}^{13} \mathrm{C}$ NMR per identificare soprattutto la posizione del gruppo ossidrilico aromatico. Il CD ha mostrato attività inibitrice 5 volte superiore ai CA e CB dell'enzima Tyrosin-Kinase che partecipa alla trasmissione dei segnali di controllo della crescita; poiché diverse Tyr-Kinases sono il prodotto di protoncogeni, inibitori di queste molecole possono rilevarsi degli importanti agenti anti-proliferativi.

La biogenesi dei clavilattoni può essere razionalizzata partendo dal geranil-idrochinone attraverso numerosi passaggi ossidativi (Fig. 10).

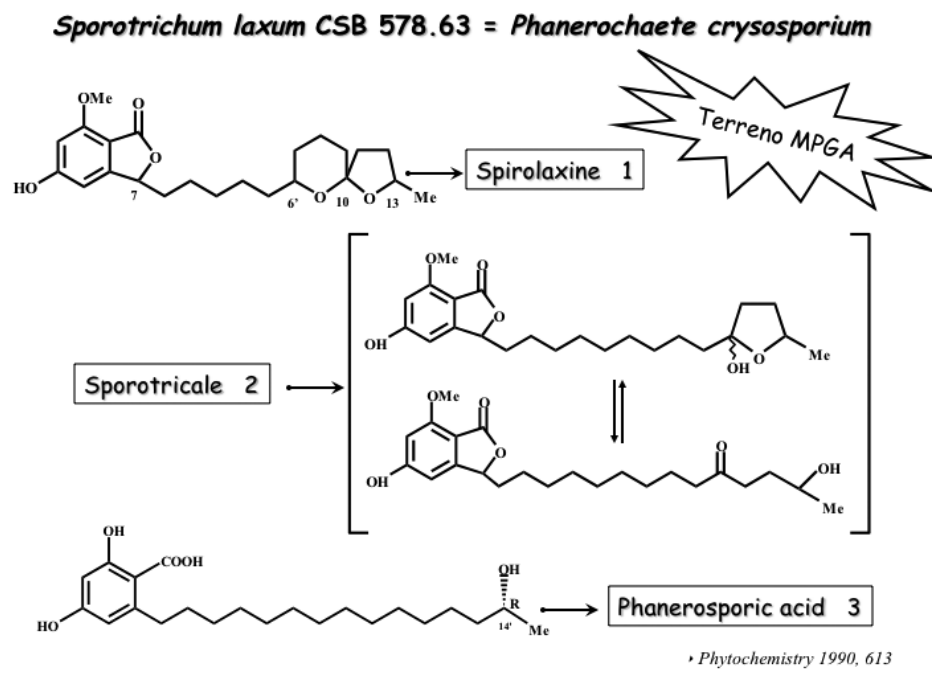

Fig. 8. 


\section{A view of the molecule of Spirolaxine 1 . showing atom - labelling scheme}<smiles>C1=CCCCC1</smiles>

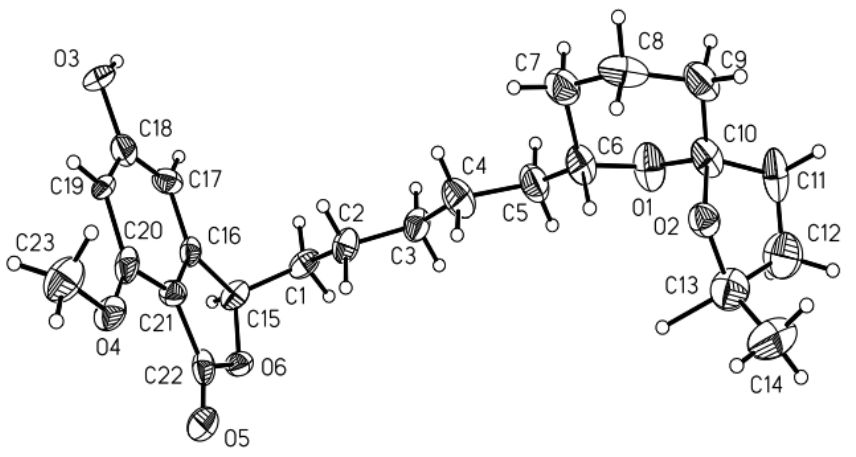

Fig. 9.

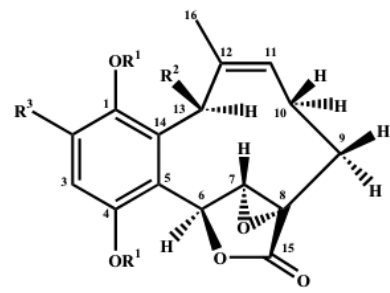

$$
\begin{aligned}
& C A: R^{1}=R^{2}=R^{3}=H \\
& C B: 1,4-\text { dioxo, } R^{2}=R^{3}=H \\
& C C: R^{1}=R^{3}=H, R^{2}=O H \\
& C D: 1,4-\text { dioxo, } R^{2}=H, R^{3}=O H \\
& C E: R^{1}=R^{3}=H, R^{2}=O M e
\end{aligned}
$$

J. Chem. Soc. Perkin 1, 1994, 2165

, Phytochemistry, 2000, 1039

, Biochemical Pharmacology, 2000, 1539

Fig. 10 . 


\section{BIBLIOGRAFIA}

[1] A. Arnone, R. Cardillo, G. Nasini, J.Chem.Soc. Perkin Trans.1, 1988, 503.

[2] A. Arnone, G. Nasini, O. Vajna de Pava, G. Assante, J.Chem.Soc. Perkin Trans.1, $1992,615$.

[3] A. Arnone, U. Brambilla, G. Nasini, O. Vajna de Pava, Tetrahedron, 1995, 48, 13357.

[4] A. Arnone, C. De Gregorio, A. Mele, G. Nasini, O. Vajna de Pava, J.Chem.Soc. Perkin Trans, 1, 2000, 745.

[5] A. Arnone, G. Candiani, G. Nasini, R. Sinisi, Tetrahedron, 2003, 59, 5033.

[6] A. Arnone, L. Merlini, G. Nasini, O. Vajna de Pava, F. Zunino, J.Chem.Soc. Perkin Trans.1, 2001, 610.

[7] L. Merlini, G. Nasini, L. Scaglioni, G. Cassinelli, C. Lanzi, Phytochemistry, 2000, $53,1039$.

[8] A. Bava, M. Clericuzio, G. Giannini, L. Malpezzi, S.V. Meille, G. Nasini, Eur.J. Org.Chem. 2005, 2292.

[9] R. Nannei, S. Dallavalle, L. Merlini, A. Bava, G. Nasini, J.Org.Chem.2006, 71, 6277, e lett. citata. 